\title{
INFRARED THERMOGRAPHY OF THE TEAR FILM IN DRY EYE
}

\author{
PHILIP B. MORGAN ${ }^{1}$, ANDREW B. TULLO ${ }^{2}$ and NATHAN EFRON ${ }^{1}$ \\ Manchester
}

\begin{abstract}
SUMMARY
Infrared ocular thermograms were recorded for a group of 36 dry eye patients and for 27 age- and sex-matched controls. Mean ocular surface temperature was greater in the dry eye group $\left(32.38 \pm 0.69{ }^{\circ} \mathrm{C}\right)$ compared with the control group $\left(31.94 \pm 0.54{ }^{\circ} \mathrm{C} ; p<0.01\right)$. In addition, there was a greater variation of temperatures across the ocular surface in the dry eye group, illustrated by the difference in temperature between the limbus and the centre of the cornea $\left(0.64 \pm 0.20^{\circ} \mathrm{C}\right.$ in dry eye patients compared with $0.41 \pm 0.20{ }^{\circ} \mathrm{C}$ in the control group; $p<0.001)$. This parameter was also shown to be greater in dry eye patients who displayed either a fast tear break-up time or a poor Schirmer's test result. Infrared thermography is a non-invasive and objective technique that may prove a useful research tool for study of the tear film, its deficiencies and its various treatment modalities.
\end{abstract}

Objective assessment of the preocular tear film in 'dry eye' is problematic. Physiological indicators such as tear osmolarity and evaporation rate, which are increased in dry eye patients, ${ }^{1,2}$ offer objectivity but are time-consuming and involve some patient inconvenience. ${ }^{3}$ Tests which are quick and simple to perform, such as the tear break-up time, Schirmer's test and ocular surface staining, can lead to unreliable and mistaken diagnoses of dry eyes. ${ }^{3,4}$ This paper analyses the potential application of infrared thermography for objective analysis of the tear film in normals and patients with dry eyes.

Infrared detection is a convenient tool for the temperature measurement of the ocular surface as it is non-invasive, instantaneous and does not require the use of anaesthetic drops. The Stefan-Boltzmann law states that the radiant emittance of an object is

From: ${ }^{1}$ Department of Optometry and Vision Sciences, University of Manchester Institute of Science and Technology, Manchester; ${ }^{2}$ Department of Ophthalmology, University of Manchester, Manchester, UK.

Correspondence to: Dr Philip B. Morgan, Department of Optometry and Vision Sciences, UMIST, PO Box 88, Manchester, M60 1QD, UK. equal to the product of the Stefan-Boltzmann constant, the emissivity of the object and the temperature of the object raised to a power of $4 .^{5}$ Therefore, the temperature of an object can be calculated if its emissivity is known and the energy emitted can be measured.

Infrared detection systems were first used for medical investigation in the late 1950 s to aid examination in breast cancer. ${ }^{6}$ They have also been employed in rheumatology, ${ }^{7,8}$ and in the investigation of deep vein thrombosis ${ }^{9}$ and headache. ${ }^{10}$

Mapstone $e^{5,11-13}$ provided the first detailed analysis of ocular temperature measured in a non-invasive manner. He established that the emissivity of the eye was $0.97-1.00 .^{5}$ Others subsequently have used more advanced instrumentation. . $^{14}$ Current infrared detection systems allow almost instantaneous and non-invasive examination with good spatial resolution. We have investigated a group of dry eye patients and a group of age- and sex-matched normals using an advanced infrared detection system.

\section{SUBJECTS AND METHODS}

Patients with dry eyes were recruited from the outpatients department of Manchester Royal Eye Hospital. Inclusion criteria were devised such that all patients were using tear replacement therapy and had either a fluorescein tear break-up time of 10 seconds or less, or a Schirmer's test result of less than $10 \mathrm{~mm}$ in 5 minutes. Moreover, patients with a history of other ocular disease, contact lens wear or facial surgery were excluded. A control group of ageand sex-matched subjects with no history or symptoms of tear film disorder was also recruited. The research followed the tenets of the Helsinki Declaration, and informed consent was obtained from all subjects after the nature and possible consequences of the study had been explained.

Infrared radiation emitted by the eye was measured with a 6T62 Thermo Tracer detection 


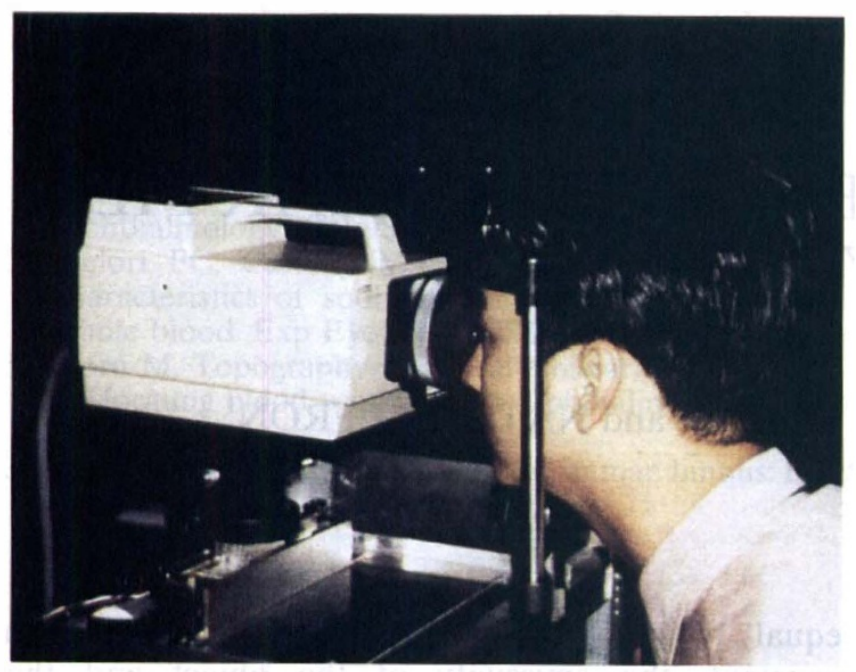

Fig. 1. The Thermo Tracer detector unit.

apparatus (NEC San-ei Instruments, Tokyo, Japan) which had been modified for ophthalmic use (Fig. 1). A detailed account of its operation has been given previously. ${ }^{18}$ The instrument is sensitive to infrared radiation between $8 \mu \mathrm{m}$ and $13 \mu \mathrm{m}$. When radiation of this wavelength is incident upon the cadmiummercury-tellurium semiconductor within the detector, there is an increase in its conductivity which can be measured. This information is interpreted by the Thermo Tracer processor unit, and displayed on a monitor in up to 8-bit colour.

Prior to thermography, each patient was confined to the laboratory for 20 minutes. Earlier work had established that this period was necessary to achieve ocular temperature stabilisation. None of the dry eye patients instilled drops on the day of examination. The Thermo Tracer recorded images at its optimum operational sensitivity $\left(0.1^{\circ} \mathrm{C}\right)$ and at a capture rate of 1.0 frame per second. Each patient had four thermograms recorded for each eye. All thermograms were recorded 4 or 5 seconds after the eyes had been closed for 3 seconds. Room temperature and humidity were measured using an electric thermohygrometer. Having been examined thermographically, each of the dry eye patients had fluorescein tear break-up time (TBUT) and the Schirmer's test undertaken by a second, masked observer.

Each ocular thermogram was recorded at a magnification of about $\times 9$. Of particular interest were five anatomical locations along the horizontal meridian running through the centre of the cornea. These were the centre of the cornea; the two intersections at the limbus; and two conjunctival sites, one temporal and one medial, each $2 \mathrm{~mm}$ from the limbus. At each of these five locations a 'temperature box', $10 \times 10$ pixels in size, was electronically interposed on the monitor screen. This represented an area of approximately $1 \mathrm{~mm}{ }^{2}$
The mean temperature of the 100 pixels enclosed within the temperature box was measured.

Both absolute and relative measures of temperature were recorded. Data from the four images recorded for each eye were pooled for these measures. The mean ocular surface temperature (MOST) was defined as the mean value of the five recorded temperature sites.

We defined one measure of relative temperature as the difference in temperature between the centre of the cornea and the mean of the two limbal measurements. This measurement was termed the 'radial temperature difference' (RTD), and represented the variation in temperature across the cornea.

\section{RESULTS}

Thirty-six dry eye patients ( 9 men, 27 women; age 58 \pm 17 years [mean $\pm \mathrm{SD}]$ ) were examined for this study. The control group was composed of 27 subjects ( 8 men, 19 women; age $57 \pm 16$ years).

Mean ocular surface temperature was greater in the dry eye group than in the controls. The values for the right eyes were $32.38 \pm 0.69^{\circ} \mathrm{C}($ mean $\pm \mathrm{SD})$ and $31.94 \pm 0.54^{\circ} \mathrm{C}$ for the dry eye group and the controls, respectively. Analysis of variance revealed this difference to be statistically significant $(p<0.01)$.

The RTD parameter was also found to be greater in the dry eye group than in the controls (for the right eye, $0.64 \pm 0.26^{\circ} \mathrm{C}$ in the dry eye group compared with $0.41 \pm 0.20^{\circ} \mathrm{C}$ in the control group; ANOVA, $p<0.001)$. This is demonstrated in Figs. 2 and 3. To facilitate further analysis, the dry eye patients were divided into smaller subgroups. Two subgroups were created for both the TBUT data and the Schirmer's test results; further subdivision was not appropriate as this would have created groups with very small numbers of patients which would not have been amenable to statistical analysis.

The thresholds for dividing the Schirmer and TBUT results were based upon the classification of dry eyes provided by Khurana et al. ${ }^{19}$ Schirmer's test scores of less than or equal to $4 \mathrm{~mm}$ were classified as Schirmer A (25 patients), and results greater than 4 $\mathrm{mm}$ as Schirmer B (10 patients). Similarly, TBUTs equal to or less than 6 seconds were classified as TBUT A (26 patients), with slower results as TBUT B (9 patients).

With the dry eye group subdivided in this manner, a two-factor analysis of variance revealed that the

Table I. Radial temperature differences for the dry eye groups

\begin{tabular}{lcc}
\hline Group & No. of patients & $\begin{array}{c}\text { RTD }\left({ }^{\circ} \mathrm{C}\right) \\
(\text { mean } \pm \text { SD) }\end{array}$ \\
\hline Schirmer $\leqslant 4 \mathrm{~mm}$ & 25 & $0.83 \pm 0.26$ \\
Schirmer $>4 \mathrm{~mm}$ & 10 & $0.55 \pm 0.28$ \\
TBUT $\leqslant 6 \mathrm{~s}$ & 26 & $0.81 \pm 0.29$ \\
TBUT $>6 \mathrm{~s}$ & 9 & $0.56 \pm 0.17$ \\
\hline
\end{tabular}




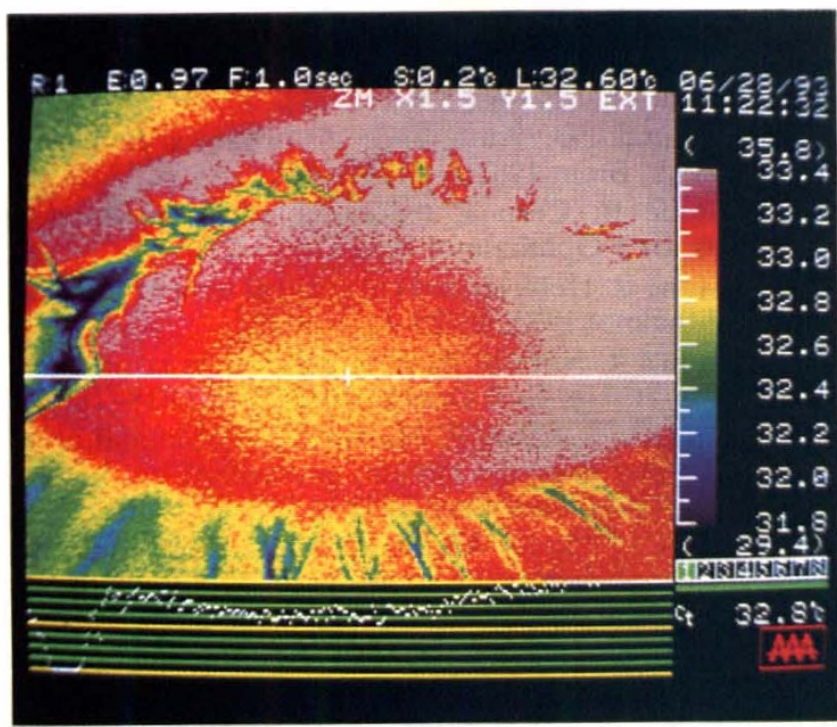

Fig. 2. Ocular thermogram of a 'normal' eye. The change of temperature across the eye is indicated graphically below the thermogram.

RTD was greater for those patients in either the Schirmer A or TBUT A groups $(p<0.01$ and $p<0.05$, respectively) (Table I). The interaction between these two factors was not significant $(p>0.05)$.

\section{DISCUSSION}

Infrared radiation between $8 \mu \mathrm{m}$ and $13 \mu \mathrm{m}$ is absorbed by water, with the degree of absorption being dependent on the thickness of the water layer. For a film of thickness $10 \mu \mathrm{m}$, the transmittance of radiation between $8 \mu \mathrm{m}$ and $13 \mu \mathrm{m}$ is approximately $30 \%$; if the thickness is $40 \mu \mathrm{m}$ or more, the transmittance approaches zero. ${ }^{20,21}$ This suggests that the exact source of the radiation which reaches the infrared detector is dependent on the thickness of the tear film. If the film is $40 \mu \mathrm{m}$ thick, as has been claimed recently, ${ }^{22}$ then all the radiation which reaches the detector must emanate from the tear film. If the film is less thick, ${ }^{23}$ there will also be a contribution from the ocular surface. In either case, it is apparent that the tear film is the principal source of radiation for measurement of this type. The temperature of the tear film will be influenced by that of the ocular surface due to local conduction processes; however, factors such as tear film evaporation and mechanics might influence the temperature when measured in this manner.

The patients from the dry eye group displayed higher ocular surface temperatures when compared with the age- and sex-matched controls. This might be explained by the increased degree of conjunctival hyperaemia associated with dry eyes. ${ }^{24,25}$ An increase in ocular temperature during inflammatory disease $\mathrm{e}^{11,18}$ and in association with bulbar conjunctival hyperaemia ${ }^{26}$ has been reported previously.

It is also important to consider the likely effect of the increase in evaporation found in dry eyes.

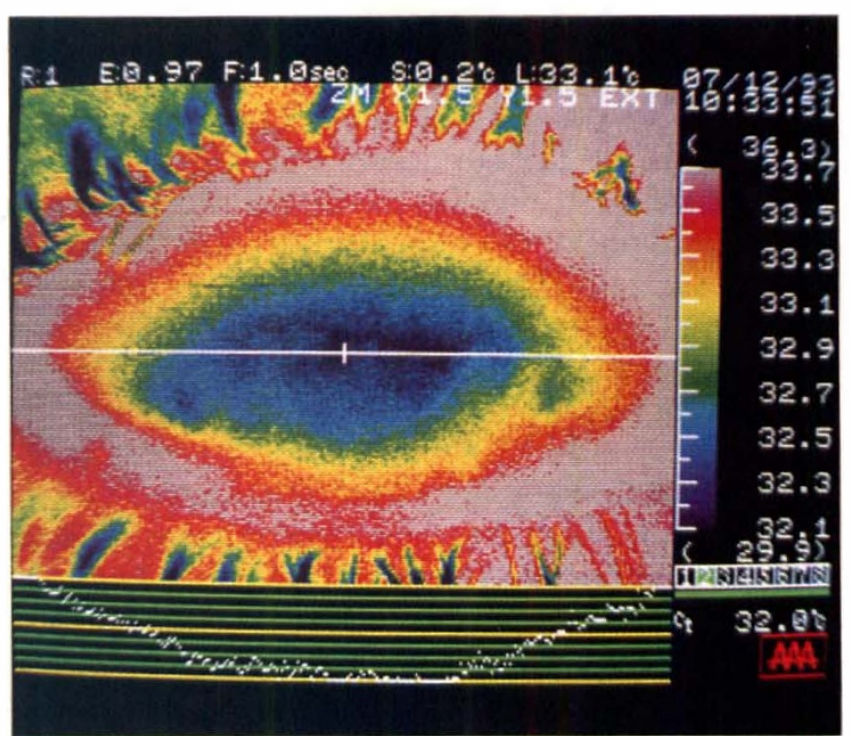

Fig. 3. Ocular thermogram of a dry eye. The change of temperature across the eye is indicated graphically below the thermogram. The temperature variation across the eye is greater than that of a normal eye.

Previous workers have measured between a twofold $^{2}$ and three-fold ${ }^{27}$ increase in evaporation rate in dry eye patients. This increase in evaporation must result in a reduction in ocular surface temperature. Rolando $\mathrm{et} \mathrm{al} .^{2}$ found that normal rate of evaporation was $4.07 \times 10^{-7} \mathrm{~g} \mathrm{~cm}^{-2} \mathrm{~s}^{-1}$ compared with $7.87 \times$ $10^{-7} \mathrm{~g} \mathrm{~cm}^{-2} \mathrm{~s}^{-1}$ in dry eyes. When this is multiplied by the latent heat of evaporation of water, $2260 \mathrm{~J}$ $\mathrm{g}^{-1}$, the amount of energy leaving the ocular surface can be derived. This is found to be $9.4 \mathrm{~W} \mathrm{~m}^{-2}$ and 18 $\mathrm{W} \mathrm{m}^{-2}$ for the controls and dry eye patients, respectively.

Scott, ${ }^{28}$ using finite element analysis to calculate the temperature distribution of the globe, found that the temperature of the ocular surface decreased by $0.24^{\circ} \mathrm{C}$ for each $20 \mathrm{~W} \mathrm{~m}^{-2}$ increase in evaporation. Thus, the data of Rolando et al. ${ }^{2}$ suggest that the ocular surface would be $0.10^{\circ} \mathrm{C}$ cooler in dry eyes due to the increased rate of evaporation. However, a net increase in temperature was demonstrated in the dry eye group. This indicates that the warming due to hyperaemia outweighs the cooling due to increased evaporation.

The RTD was greater in the dry eye group than in the controls. Moreover, it was shown to be greater in severe dry eyes (in either Schirmer A or TBUT A categories) when compared with moderate dry eyes (Schirmer B or TBUT B categories).

It is possible to explain this observation with a qualitative model. This model assumes that the tear film acts as a distributor of heat which serves to reduce the variation in temperature across the ocular surface. If an eye without a tear film could be examined thermographically, the difference in temperature between the centre of the cornea and the limbus would be relatively great, due to the 
difference in vasculature between these sites. If, at the other extreme, an eye with an extremely thick tear film were to be examined, the variation in temperature at the surface of the film would be almost eliminated, because the action of the tear film would be to 'mask' the temperature variations at the corneal surface. This model predicts that there will be greater temperature variation with a thin tear film (in the case of a severe dry eye) than with a thicker tear film (in both a normal tear film or less severe dry eyes).

Although thermography currently requires costly equipment, it offers the objectivity of measurements such as evaporation rate and osmolarity with the convenience of Schirmer's test and TBUT. This form of ocular examination is suitable for the examination of the tear film, and the investigation of the various modalities of dry eye treatment.

The authors thank Professor David McLeod for his valuable comments on the manuscript. This work was supported by grants from Polymer Technology, Inc., Wilmington, Mass., USA, and from the Research Endowment Fund of Manchester Royal Eye Hospital.

Key words: Dry eye, Infrared, Tear film, Temperature, Thermography.

\section{REFERENCES}

1. Gilbard JP. Tear film osmolarity and keratoconjunctivitis sicca. In: Holly F, editor. The preocular tear film in health, disease and contact lens wear. Lubbock, Tx: Dry Eye Institute, 1986:127-39.

2. Rolando M, Refojo MF, Kenyon KR. Increased tear evaporation in eyes with keratoconjunctivitis sicca. Arch Ophthalmol 1983;101:557-8.

3. Seal DV, Mackie IA. The questionable dry eye as a clinical and biochemical entity. In: Holly F, editor. The preocular tear film in health, disease and contact lens wear. Lubbock, Tx: Dry Eye Institute, 1986:41-51.

4. Paschides CA, Kitsios G, Karakostas KX, Psillas C, Moutsopoulos HM. Evaluation of tear break-up time, Schirmer's-I test and rose bengal staining as confirmatory tests for keratoconjunctivitis sicca. Clin Exp Rheumatol 1989;7:155-7.

5. Mapstone R. Measurement of corneal temperature. Exp Eye Res 1968;7:237-43.

6. Lawson RN, Chughtai MS. Breast cancer and body temperature. Can Med Assoc J 1963;88:68-70.

7. Darton K, Black CM. The use of infra-red thermography in a rheumatology unit. Br J Rheumatol 1990; 29:291-2.

8. Boas NF. Thermography in rheumatoid arthritis. Ann NY Acad Sci 1964;121:223-34.
9. Cooke ED. Thermography. In: Nicolaides AN, Yao T, editors. Investigation of vascular disorders. New York: Churchill Livingstone, 1981:416-42.

10. Drummond PD. Autonomic disturbances in cluster headache. Brain 1988;111:1199-209.

11. Mapstone R. Corneal thermal patterns in anterior uveitis. Br J Ophthalmol 1968;52:917-21.

12. Mapstone R. Determinants of corneal temperature. $\mathrm{Br}$ J Ophthalmol 1968;52:729-41.

13. Mapstone R. Normal thermal patterns in cornea and periorbital skin. Br J Ophthalmol 1968;52:818-27.

14. Rosenstock T, Chart P, Hurwitz JJ. Inflammation of the lacrimal drainage system: assessment by thermography. Ophthalmic Surg 1983;14:229-37.

15. Fielder AR, Winder AF, Sheraidah GAK, Cooke ED. Problems with corneal arcus. Trans Ophthalmol Soc UK 1981;101:22-6.

16. Efron N, Young G, Brennan NA. Ocular surface temperature. Curr Eye Res 1989;8:901-6.

17. Alio J, Padron M. Normal variations in the thermographic pattern of the orbito-ocular region. Diagn Imaging 1982;51:93-8.

18. Morgan PB, Soh MP, Efron N, Tullo AB. Potential applications of ocular thermography. Optom Vis Sci 1993;70:568-76.

19. Khurana AK, Chaudhary R, Ahluwalia BK, Gupta S. Tear film profile in dry eye. Acta Ophthalmol (Copenh) 1991;69:79-86.

20. Plyler EK, Aquista N. IR absorption of liquid water from 2 to $42 \mu$. J Opt Soc Am 1954;44:515.

21. Hamano H, Minami S, Sugimori Y. Experiments in thermometry of the anterior portion of the eye wearing a contact lens by means of infrared thermometer. Contacto 1969;13:12-22.

22. Prydal JI, Artal P, Woon H, Campbell FW. Study of human precorneal tear film thickness and structure using laser interferometry. Invest Ophthalmol Vis Sci 1992;33:2006-11.

23. Holly FJ. Formation and rupture of the tear film. Exp Eye Res 1973;15:515-25.

24. Hunter PA, Watson PG. Allergic eye diseases: episcleritis and scleritis. In: Spalton DJ, Hitchings RA, Hunter PA, editors. Atlas of clinical ophthalmology. Edinburgh: Churchill Livingstone, 1984.

25. Golding TR. Evaluation of tear film impairment in dry eye and hydrophilic contact lens wear. $\mathrm{PhD}$ thesis. University of Melbourne, 1992.

26. Efron N, Brennan NA, Hore J, Rieper K. Temperature of the hyperemic bulbar conjunctiva. Curr Eye Res 1988;7:615-8.

27. Mathers WD, Binarao G, Petroll M. Ocular water evaporation and the dry eye: a new measuring device. Cornea 1993;12:335-40.

28. Scott JA. A finite element model of heat transport in the human eye. Phys Med Biol 1988;33:227-41. 